General Mathematics Vol. 27, No. 1 (2019), 29-36

DOI:10.2478/gm-2019-0003

sciendo

\title{
Properties of the intermediate point from a mean value theorem of the integral calculus - II ${ }^{1}$
}

\author{
Emilia-Loredana Pop, Dorel Duca, Augusta Raţiu
}

\begin{abstract}
In this paper we consider two continuous functions $f, g:[a, b] \rightarrow \mathbb{R}$ and we study for these ones, under which circumstances the intermediate point function is four order differentiable at the point $x=a$ and we calculate its derivative.
\end{abstract}

2010 Mathematics Subject Classification: 26A24, 11H60.

Key words and phrases: intermediate point, mean-value theorem.

\section{Introduction and Preliminaries}

We start by considering the discussions related to the second mean value theorem of the integral calculus (or Bonnet theorem) and also, the idea from the paper [7] and some results from the paper [2].

Let us consider two continuous functions $f, g:[a, b] \rightarrow \mathbb{R}$ with the properties:

(a) the function $f$ is decreasing on $[a, b]$,

(b) $f(x) \geq 0$, for all $x \in[a, b]$,

then, for each $x \in(a, b]$, there exists a point $c_{x} \in[a, x]$ such that

$$
\int_{a}^{x} f(t) g(t) \mathrm{d} t=f(a) \int_{a}^{c_{x}} g(t) \mathrm{d} t .
$$

If for each $x \in(a, b]$ we choose one $c_{x} \in[a, x]$ such that (1) holds, we can define the function $c:(a, b] \rightarrow[a, b]$ by

$$
c(x)=c_{x}, \text { for all } x \in(a, b],
$$

with the property

$$
\int_{a}^{x} f(t) g(t) \mathrm{d} t=f(a) \int_{a}^{c(x)} g(t) \mathrm{d} t, \text { for all } x \in(a, b] .
$$

Moreover, from ([7]) we have that

\footnotetext{
${ }^{1}$ Received 27 March, 2019

Accepted for publication (in revised form) 8 May, 2019
} 
Theorem 1 Let $a, b \in \mathbb{R}$ with $a<$ b.If the continuous functions $f, g:[a, b] \rightarrow \mathbb{R}$ satisfies properties:

(a) the function $f$ is decreasing on $[a, b]$,

(b) $f(x) \geq 0$, for all $x \in[a, b]$,

then there exists a function $c:(a, b] \rightarrow[a, b]$ such that (3) holds.

When $x \in(a, b]$ tends to $a$, because $|c(x)-a| \leq|x-a|$, we have

$$
\lim _{x \rightarrow a} c(x)=a .
$$

Then the function $\bar{c}:[a, b] \rightarrow[a, b]$ defined by

$$
\bar{c}(x)= \begin{cases}c(x), & \text { if } x \in(a, b] \\ a, & \text { if } x=a .\end{cases}
$$

is continuous at $x=a$.

For $x \in(a, b]$, we have

$$
\frac{\bar{c}(x)-\bar{c}(a)}{x-a}=\frac{c(x)-a}{x-a} .
$$

If we denote by

$$
\theta(x)=\frac{c(x)-a}{x-a},
$$

then

$$
\theta(x) \in(0,1)
$$

and

$$
c(x)=a+(x-a) \theta(x)
$$

and hence

$$
\int_{a}^{x} f(t) g(t) \mathrm{d} t=f(a) \int_{a}^{a+(x-a) \theta(x)} g(t) \mathrm{d} t, \text { for all } x \in(a, b] .
$$

Hence, the following result is true.

Theorem 2 (see [7]) Let $a, b \in \mathbb{R}$ with $a<b$.If the continuous functions $f, g$ : $[a, b] \rightarrow \mathbb{R}$ satisfies properties:

(a) the function $f$ is decreasing on $[a, b]$,

(b) $f(x) \geq 0$, for all $x \in[a, b]$,

then there exists a function $\theta:(a, b] \rightarrow[0,1]$ such that (4) holds.

If $F$ be is a primitive of the function $f g$ and $G$ is a primitive of the function $g$, then, from the Leibniz Newton Theorem, the equality (4) becomes

$$
F(x)-F(a)=f(a)[G(a+(x-a) \theta(x))-G(a)] \text {, for all } x \in(a, b] .
$$

In the paper [7], we had proved the following theorems. 
Theorem 3 (see [7]) Let $a, b \in \mathbb{R}$ with $a<b$ and let $f, g$ be two continuous functions on $[a, b]$. If

(a) the function $f$ is decreasing on $[a, b]$,

(b) $f(x) \geq 0$, for all $x \in[a, b]$,

(c) $f(a) g(a) \neq 0$, then

$1^{\circ}$ The function $\theta:(a, b] \rightarrow[0,1]$ has limit at the point $x=a$ and

$$
\lim _{x \rightarrow a} \theta(x)=1 .
$$

$2^{\circ}$ The function $\bar{c}$ is derivable at $a$ and

$$
\bar{c}^{\prime}(a)=1 .
$$

Let $\bar{\theta}:[a, b] \rightarrow[0,1]$ the function defined by

$$
\bar{\theta}(x)= \begin{cases}\theta(x), & \text { if } x \in(a, b] \\ 1, & \text { if } x=a .\end{cases}
$$

Theorem 4 (see [7]) Let $a, b \in \mathbb{R}$ with $a<b$ and let $f, g$ be two differentiable functions on $[a, b]$. If

(a) the function $f$ is decreasing on $[a, b]$,

(b) $f(x) \geq 0$, for all $x \in[a, b]$,

(c) $f^{\prime}$ and $g^{\prime}$ are continuous at $x=a$,

(d) $f(a) g(a) \neq 0$,

then

$1^{\circ}$ The function $\bar{\theta}$ is differentiable at $x=a$ and

$$
\bar{\theta}^{\prime}(a)=\frac{f^{\prime}(a)}{2 f(a)} .
$$

$2^{\circ}$ The function $\bar{c}$ is twice differentiable at $x=a$ and

$$
\bar{c}^{\prime \prime}(a)=\frac{f^{\prime}(a)}{f(a)} .
$$

Theorem 5 (see [7]) Let $a, b \in \mathbb{R}$ with $a<b$ and let $f, g$ be two twice differentiable functions on $[a, b]$. If

(a) the function $f$ is decreasing on $[a, b]$,

(b) $f(x) \geq 0$, for all $x \in[a, b]$,

(c) $f^{\prime \prime}$ and $g^{\prime \prime}$ are continuous at $x=a$,

(d) $f(a) g(a) \neq 0$,

then

$1^{\circ}$ The function $\bar{\theta}$ is twice differentiable at $x=a$ and

$$
\bar{\theta}^{\prime \prime}(a)=\frac{f^{\prime \prime}(a) g(a)-f^{\prime}(a) g^{\prime}(a)}{3 f(a) g(a)} .
$$


$2^{\circ}$ The function $\bar{c}$ is third differentiable at $x=a$ and

$$
\bar{c}^{\prime \prime \prime}(a)=\frac{f^{\prime \prime}(a) g(a)-f^{\prime}(a) g^{\prime}(a)}{f(a) g(a)} .
$$

The purpose of this paper is to establish under which circumstances the function $\bar{c}$ is the four order differentiable at the point $x=a$ and to compute its derivative $\bar{c}^{(4)}(a)$. Do the derivative $\bar{c}^{(4)}(a)$ depend upon the functions $f$ and $g$ ? If there exist several functions $\bar{c}$ which satisfy (3), do the derivative of the function $\bar{c}$ at $x=a$ depend upon the function $\bar{c}$ we choose?

These types of discussions, related to the differentiability of the functions and not only, to which the derivatives have been calculated, was also considered in papers like [1], [3], [4], [5], [6], [8].

\section{Main results}

Theorem 6 Let $a, b \in \mathbb{R}$ with $a<b$ and let $f, g$ be two four times differentiable functions on $[a, b]$. If

(a) the function $f$ is decreasing on $[a, b]$,

(b) $f(x) \geq 0$, for all $x \in[a, b]$,

(c) $f^{\prime \prime \prime}$ and $g^{\prime \prime \prime}$ are continuous at $x=a$, then

(d) $f(a) g(a) \neq 0$,

$1^{\circ}$ The function $\bar{\theta}$ is three times differentiable at $x=a$ and

$$
\begin{gathered}
\bar{\theta}^{\prime \prime \prime}(a)= \\
=\frac{1}{(2 f(a) g(a))^{2}}\left[4 f(a) f^{\prime}(a)\left(g^{\prime}(a)\right)^{2}-3\left(f^{\prime}(a)\right)^{2} g(a) g^{\prime}(a)-\right. \\
\left.-f(a) f^{\prime \prime}(a) g(a) g^{\prime}(a)-3 f(a) f^{\prime}(a) g(a) g^{\prime \prime}(a)+f(a) f^{\prime \prime \prime}(a) g^{2}(a)\right] .
\end{gathered}
$$

$2^{\circ}$ The function $\bar{c}$ is four differentiable at $x=a$ and

$$
\begin{gathered}
\bar{c}^{i v}(a)= \\
=\frac{1}{(f(a) g(a))^{2}}\left[4 f(a) f^{\prime}(a)\left(g^{\prime}(a)\right)^{2}-3\left(f^{\prime}(a)\right)^{2} g(a) g^{\prime}(a)-\right. \\
\left.-f(a) f^{\prime \prime}(a) g(a) g^{\prime}(a)-3 f(a) f^{\prime}(a) g(a) g^{\prime \prime}(a)+f(a) f^{\prime \prime \prime}(a) g^{2}(a)\right] .
\end{gathered}
$$

Proof. $1^{\circ}$ By Taylor's theorem, for each $x \in(a, b]$, there are $\xi_{x}, \eta_{x} \in(a, x)$ such that

$$
\begin{aligned}
& F(x)=F(a)+\frac{F^{\prime}(a)}{1 !}(x-a)+\frac{F^{\prime \prime}(a)}{2 !}(x-a)^{2}+\frac{F^{\prime \prime \prime}(a)}{3 !}(x-a)^{3}+\frac{F^{i v}\left(\xi_{x}\right)}{4 !}(x-a)^{4} \\
& G(x)=G(a)+\frac{G^{\prime}(a)}{1 !}(x-a)+\frac{G^{\prime \prime}(a)}{2 !}(x-a)^{2}+\frac{G^{\prime \prime \prime}(a)}{3 !}(x-a)^{3}+\frac{G^{i v}\left(\eta_{x}\right)}{4 !}(x-a)^{4}
\end{aligned}
$$


We replace these formulas in (5) and divide by $(x-a)$ get

$$
\begin{aligned}
& \frac{F^{\prime}(a)}{1 !}+\frac{F^{\prime \prime}(a)}{2 !}(x-a)+\frac{F^{\prime \prime \prime}(a)}{3 !}(x-a)^{2}+\frac{F^{i v}\left(\xi_{x}\right)}{4 !}(x-a)^{3}= \\
& =f(a)\left[\frac{G^{\prime}(a)}{1 !} \theta(x)+\frac{G^{\prime \prime}(a)}{2 !}(x-a) \theta^{2}(x)+\frac{G^{\prime \prime \prime}(a)}{3 !}(x-a)^{2} \theta^{3}(x)+\frac{G^{i v}\left(\eta_{x}\right)}{4 !}(x-a)^{3} \theta^{4}(x)\right] .
\end{aligned}
$$

For each $x \in[a, b]$, we have

$$
\begin{gathered}
F(x)=\int_{a}^{x} f(t) g(t) d t, F^{\prime}(x)=f(x) g(x), \\
F^{\prime \prime}(x)=f^{\prime}(x) g(x)+f(x) g^{\prime}(x), \\
F^{\prime \prime \prime}(x)=f^{\prime \prime}(x) g(x)+2 f^{\prime}(x) g^{\prime}(x)+f(x) g^{\prime \prime}(x), \\
F^{i v}(x)=f^{\prime \prime \prime}(x) g(x)+3 f^{\prime \prime}(x) g^{\prime}(x)+3 f^{\prime}(x) g^{\prime \prime}(x)+f(x) g^{\prime \prime \prime}(x), \\
G(x)=\int_{a}^{x} g(t) d t, \quad G^{\prime}(x)=g(x), \quad G^{\prime \prime}(x)=g^{\prime}(x), \\
G^{\prime \prime \prime}(x)=g^{\prime \prime}(x), \quad G^{i v}(x)=g^{\prime \prime \prime}(x),
\end{gathered}
$$

so the last equality becomes

$$
\begin{gathered}
f(a) g(a)[\theta(x)-1]= \\
=\frac{1}{2 !}\left[f^{\prime}(a) g(a)+f(a) g^{\prime}(a)-f(a) g^{\prime}(a) \theta^{2}(x)\right](x-a)+ \\
+\frac{1}{3 !}\left[f^{\prime \prime}(a) g(a)+2 f^{\prime}(a) g^{\prime}(a)+f(a) g^{\prime \prime}(a)-f(a) g^{\prime \prime}(a) \theta^{3}(x)\right](x-a)^{2}+ \\
+\frac{1}{4 !}\left[f^{\prime \prime \prime}\left(\xi_{x}\right) g\left(\xi_{x}\right)+3 f^{\prime \prime}\left(\xi_{x}\right) g^{\prime}\left(\xi_{x}\right)+3 f^{\prime}\left(\xi_{x}\right) g^{\prime \prime}\left(\xi_{x}\right)+\right. \\
\left.+f\left(\xi_{x}\right) g^{\prime \prime \prime}\left(\xi_{x}\right)-f(a) g^{\prime \prime \prime}\left(\eta_{x}\right) \theta^{4}(x)\right](x-a)^{3} .
\end{gathered}
$$

In relation (6) we let $x \rightarrow a$ and obtain

$$
\lim _{x \rightarrow a} \theta(x)=1 .
$$

When $x \in I \backslash\{a\}$, we divide relation (6) by $(x-a)$ and get

$$
\begin{gathered}
\frac{\theta(x)-1}{x-a}= \\
\frac{1}{2 ! f(a) g(a)}\left[f^{\prime}(a) g(a)+f(a) g^{\prime}(a)-f(a) g^{\prime}(a) \theta^{2}(x)\right]+
\end{gathered}
$$




$$
\begin{gathered}
+\frac{1}{3 ! f(a) g(a)}\left[f^{\prime \prime}(a) g(a)+2 f^{\prime}(a) g^{\prime}(a)+f(a) g^{\prime \prime}(a)-f(a) g^{\prime \prime}(a) \theta^{3}(x)\right](x-a)+ \\
+\frac{1}{4 ! f(a) g(a)}\left[f^{\prime \prime \prime}\left(\xi_{x}\right) g\left(\xi_{x}\right)+3 f^{\prime \prime}\left(\xi_{x}\right) g^{\prime}\left(\xi_{x}\right)+3 f^{\prime}\left(\xi_{x}\right) g^{\prime \prime}\left(\xi_{x}\right)+\right. \\
\left.+f\left(\xi_{x}\right) g^{\prime \prime \prime}\left(\xi_{x}\right)-f(a) g^{\prime \prime \prime}\left(\eta_{x}\right) \theta^{4}(x)\right](x-a)^{2} .
\end{gathered}
$$

In relation (7) we let $x \rightarrow a$ and obtain

$$
\bar{\theta}^{\prime}(a)=\lim _{x \rightarrow a} \frac{\theta(x)-1}{x-a}=\frac{f^{\prime}(a)}{2 f(a)} .
$$

Next, we rewrite relation (7) as,

$$
\begin{gathered}
\frac{\theta(x)-1}{x-a}-\bar{\theta}^{\prime}(a)= \\
=-\frac{f^{\prime}(a)}{2 f(a)}+\frac{1}{2 ! f(a) g(a)}\left[f^{\prime}(a) g(a)+f(a) g^{\prime}(a)-f(a) g^{\prime}(a) \theta^{2}(x)\right]+ \\
+\frac{1}{3 ! f(a) g(a)}\left[f^{\prime \prime}(a) g(a)+2 f^{\prime}(a) g^{\prime}(a)+f(a) g^{\prime \prime}(a)-f(a) g^{\prime \prime}(a) \theta^{3}(x)\right](x-a)+ \\
+\frac{1}{4 ! f(a) g(a)}\left[f^{\prime \prime \prime}\left(\xi_{x}\right) g\left(\xi_{x}\right)+3 f^{\prime \prime}\left(\xi_{x}\right) g^{\prime}\left(\xi_{x}\right)+3 f^{\prime}\left(\xi_{x}\right) g^{\prime \prime}\left(\xi_{x}\right)+\right. \\
\left.+f\left(\xi_{x}\right) g^{\prime \prime \prime}\left(\xi_{x}\right)-f(a) g^{\prime \prime \prime}\left(\eta_{x}\right) \theta^{4}(x)\right](x-a)^{2} .
\end{gathered}
$$

or, equivalently,

$$
\begin{gathered}
\frac{\frac{\theta(x)-1}{x-a}-\bar{\theta}^{\prime}(a)}{x-a}= \\
=\frac{1}{2 ! f(a) g(a)} \frac{f(a) g^{\prime}(a)-f(a) g^{\prime}(a) \theta^{2}(x)}{x-a}+ \\
+\frac{1}{3 ! f(a) g(a)}\left[f^{\prime \prime}(a) g(a)+2 f^{\prime}(a) g^{\prime}(a)+f(a) g^{\prime \prime}(a)-f(a) g^{\prime \prime}(a) \theta^{3}(x)\right]+ \\
+\frac{1}{4 ! f(a) g(a)}\left[f^{\prime \prime \prime}\left(\xi_{x}\right) g\left(\xi_{x}\right)+3 f^{\prime \prime}\left(\xi_{x}\right) g^{\prime}\left(\xi_{x}\right)+3 f^{\prime}\left(\xi_{x}\right) g^{\prime \prime}\left(\xi_{x}\right)+\right. \\
\left.+f\left(\xi_{x}\right) g^{\prime \prime \prime}\left(\xi_{x}\right)-f(a) g^{\prime \prime \prime}\left(\eta_{x}\right) \theta^{4}(x)\right](x-a)
\end{gathered}
$$

From here result that

$$
\bar{\theta}^{\prime \prime}(a)=\frac{f^{\prime \prime}(a) g(a)-f^{\prime}(a) g^{\prime}(a)}{3 f(a) g(a)} .
$$


From relation (8) we obtain

$$
\begin{gathered}
\frac{\frac{\theta(x)-1}{x-a}-\bar{\theta}^{\prime}(a)}{x-a}-\frac{\bar{\theta}^{\prime \prime}(a)}{2 !}= \\
=-\frac{f(a) g^{\prime}(a)}{2 ! f(a) g(a)} \frac{\theta(x)-1}{x-a}[\theta(x)+1]-\frac{f^{\prime \prime}(a) g(a)-f^{\prime}(a) g^{\prime}(a)}{3 ! f(a) g(a)}+ \\
+\frac{1}{3 ! f(a) g(a)}\left[f^{\prime \prime}(a) g(a)+2 f^{\prime}(a) g^{\prime}(a)+f(a) g^{\prime \prime}(a)-f(a) g^{\prime \prime}(a) \theta^{3}(x)\right]+ \\
+\frac{1}{4 ! f(a) g(a)}\left[f^{\prime \prime \prime}\left(\xi_{x}\right) g\left(\xi_{x}\right)+3 f^{\prime \prime}\left(\xi_{x}\right) g^{\prime}\left(\xi_{x}\right)+3 f^{\prime}\left(\xi_{x}\right) g^{\prime \prime}\left(\xi_{x}\right)+\right. \\
\left.+f\left(\xi_{x}\right) g^{\prime \prime \prime}\left(\xi_{x}\right)-f(a) g^{\prime \prime \prime}\left(\eta_{x}\right) \theta^{4}(x)\right](x-a) .
\end{gathered}
$$

Here we divide by $x-a$, where $x \neq a$, and we have

$$
\begin{aligned}
& \frac{\frac{\frac{\theta(x)-1}{x-a}-\bar{\theta}^{\prime}(a)}{x-a}-\frac{\bar{\theta}^{\prime \prime}(a)}{2 !}}{x-a}= \\
& =\frac{1}{3 ! f(a) g(a)} \frac{3 f^{\prime}(a) g^{\prime}(a)-f(a) g^{\prime \prime}(a)\left[\theta^{3}(x)-1\right]-3 f(a) g^{\prime}(a) \frac{\theta^{2}(x)-1}{x-a}}{x-a}+ \\
& +\frac{1}{4 ! f(a) g(a)}\left[f^{\prime \prime \prime}\left(\xi_{x}\right) g\left(\xi_{x}\right)+3 f^{\prime \prime}\left(\xi_{x}\right) g^{\prime}\left(\xi_{x}\right)+3 f^{\prime}\left(\xi_{x}\right) g^{\prime \prime}\left(\xi_{x}\right)+\right. \\
& \left.+f\left(\xi_{x}\right) g^{\prime \prime \prime}\left(\xi_{x}\right)-f(a) g^{\prime \prime \prime}\left(\eta_{x}\right) \theta^{4}(x)\right] .
\end{aligned}
$$

Now, we let $x \rightarrow a$, and get

$$
\begin{gathered}
\bar{\theta}^{\prime \prime \prime}(a)= \\
=\frac{1}{(2 f(a) g(a))^{2}}\left[4 f(a) f^{\prime}(a)\left(g^{\prime}(a)\right)^{2}-3\left(f^{\prime}(a)\right)^{2} g(a) g^{\prime}(a)-\right. \\
\left.-f(a) f^{\prime \prime}(a) g(a) g^{\prime}(a)-3 f(a) f^{\prime}(a) g(a) g^{\prime \prime}(a)+f(a) f^{\prime \prime \prime}(a) g^{2}(a)\right] .
\end{gathered}
$$

$2^{\circ}$ Follows immediately from $1^{\circ}$.

\section{Conclusions and further challenges}

In this paper we introduced conditions for the functions $f$ and $g$ such that the intermediate point of functions $\bar{c}$ and $\bar{\theta}$ to be derivable in the point $a$ and we provided the derivative $\bar{\theta}^{\prime \prime \prime}(a)$ and $\bar{c}^{i v}(a)$.

In future we want to see in which conditions the functions $\bar{c}$ and $\bar{\theta}$ are derivable of order $n$ in the point $a$ and to calculate the corresponding derivative. 


\section{References}

[1] D. I. Duca, Analiză Matematică, Casa Cărţii de Ştiinţă, Cluj-Napoca, vol. 1, 2013.

[2] D. I. Duca, Analiză Matematică, vol. 2, to appear.

[3] D. I. Duca, A Note on the Mean Value Theorem, Didactica Matematica, vol. 19, 2003, 91-102.

[4] D. I. Duca, Properties of the intermediate point from the Taylor's theorem, Mathematical Inequalities and Applications, vol. 12, no. 4, 2009, 763-771.

[5] D. I. Duca, E. Duca, Exerciţii şi probleme de analiză matematică, Casa Cărţii de Ştiinţă, vol. 2, 2009.

[6] D. I. Duca, O. Pop, Concerning the Intermediate Point in the Mean-Value Theorem, Mathematical Inequalities \& Applications, vol. 12, no. 3, 2009, 499512.

[7] E.-L. Pop, D. I. Duca, A. Ratiu, Properties of the intermediate point from a mean value theorem of the integral calculus, General Mathematics, vol. 25, no. $1-2,2017,3-10$.

[8] T. Trif, Asymptotic Behavior of Intermediate Points in certain Mean Value Theorems, Journal of Mathematical Inequalities, vol. 2, no. 2, 2008, 151-161.

\section{Emilia-Loredana Pop}

Babeş-Bolyai University

Faculty of Mathematics and Computer Science

Department of Mathematics

Street Mihail Kogălniceanu 1, 400084 Cluj-Napoca, România

e-mail: pop_emilia_loredana@yahoo.com

\section{Dorel Duca}

Babeş-Bolyai University

Faculty of Mathematics and Computer Science

Department of Mathematics

Street Mihail Kogălniceanu 1, 400084 Cluj-Napoca, România

e-mail: dorelduca@yahoo.com

\section{Augusta Raţiu}

Lucian Blaga University of Sibiu

Faculty of Science

Department of Mathematics and Computer Science

Street Dr. I. Raţiu 5-7, 550012 Sibiu, Romania

e-mail: augu2003@yahoo.com 